\title{
A short and efficient synthesis of (+)-totarol
}

\author{
Victor Rogachev, ${ }^{a}$ Thorsten Löhl, ${ }^{\mathrm{b}}$ Thomas Markert, ${ }^{\mathrm{b}}$ and Peter Metz*a \\ ${ }^{a}$ Fachrichtung Chemie und Lebensmittelchemie, Organische Chemie I, \\ Technische Universität Dresden, Bergstrasse 66, D-01069 Dresden, Germany \\ ${ }^{b}$ Cognis GmbH, Henkelstraße 67, D-40589 Düsseldorf, Germany \\ E-mail:peter.metz@chemie.tu-dresden.de
}

Dedicated to Professor Rainer Beckert on the occasion of his $60^{\text {th }}$ birthday

\begin{abstract}
A concise route to multigram quantities of the antibacterial diterpene (+)-totarol (1) is reported. (-)-Sclareol (2) was converted to the target compound 1 using either a six- or a seven-step sequence, while only three steps were required to access (+)-totarol (1) starting from (+)-manool (9) or (+)-13-epi-manool (10), respectively. A novel one-pot intramolecular aldol condensation/ $\alpha$-alkylation protocol served as the key operation for streamlining the syntheses of $\mathbf{1}$.
\end{abstract}

Keywords: Antibacterials, diterpenes, manool, natural products, sclareol, semisynthesis

\section{Introduction}

The tricyclic diterpene (+)-totarol (1) has been isolated from many plants, with the heartwood of the New Zealand native tree Podocarpus totara being the most abundant source (Figure 1). ${ }^{1}$

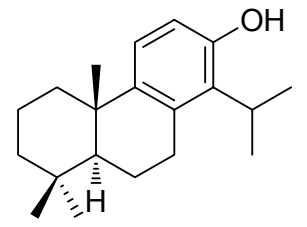

1

Figure 1. Structure of the bioactive diterpene (+)-totarol (1).

(+)-Totarol (1) has been shown to display a range of interesting bioactivities. ${ }^{1}$ Thus, it is an antistaphylococcal antimicrobial agent, ${ }^{2}$ and it is likely to inhibit bacterial cytokinesis by targeting the protein FtsZ, which may be useful for the development of novel antitubercular drugs. ${ }^{3}$ Furthermore, it compromises the functional integrity of phospholipid membranes, ${ }^{4}$ acts as 
an inhibitor of the bacterial respiratory chain, ${ }^{5}$ and shows marked antiplasmodial activity against Plasmodium falciparum ${ }^{6}$ and potent bactericidal activity against Propionibacterium acnes, ${ }^{7}$ which causes skin diseases.

The first synthesis of racemic totarol that also confirmed its relative configuration succeeded in 1958 commencing with 2,2,6-trimethylcyclohexanone, ${ }^{8}$ while the first enantioselective synthesis of $(+)$-totarol (1) was disclosed in 1979 utilizing $(R)-(-)$ - $\alpha$-cyclocitral as the starting material. ${ }^{9}$ A more recent synthesis of racemic totarol applied a polyolefin cyclization for generation of the tricyclic skeleton, ${ }^{10}$ and further enantioselective syntheses of (+)-totarol (1) were achieved from zamoranic acid, ${ }^{11}$ through a chemoenzymatic approach, ${ }^{12}$ and by epoxide cyclization. ${ }^{13}$ Furthermore, the total synthesis of racemic totarol methyl ether from a naphthalene derivative $^{14}$ and the synthesis of totarol analogs from manool along with an investigation of their antibacterial activities were published. ${ }^{15}$

Since the reported syntheses of (+)-totarol (1) required rather lengthy reaction sequences, we developed concise routes to enantiomerically pure 1 from (-)-sclareol (2) that efficiently provided multigram amounts of this diterpene using only six or seven steps. A novel one-pot intramolecular aldol condensation/ $\alpha$-alkylation protocol served as the crucial operation for streamlining these syntheses, which also allowed a three-step conversion of (+)-manool (9) or (+)-13-epi-manool (10), respectively, to (+)-totarol (1). ${ }^{16}$

\section{Results and Discussion}

Preparation of the key intermediate $7^{17}$ from (-)-sclareol (2) is depicted in Scheme 1.
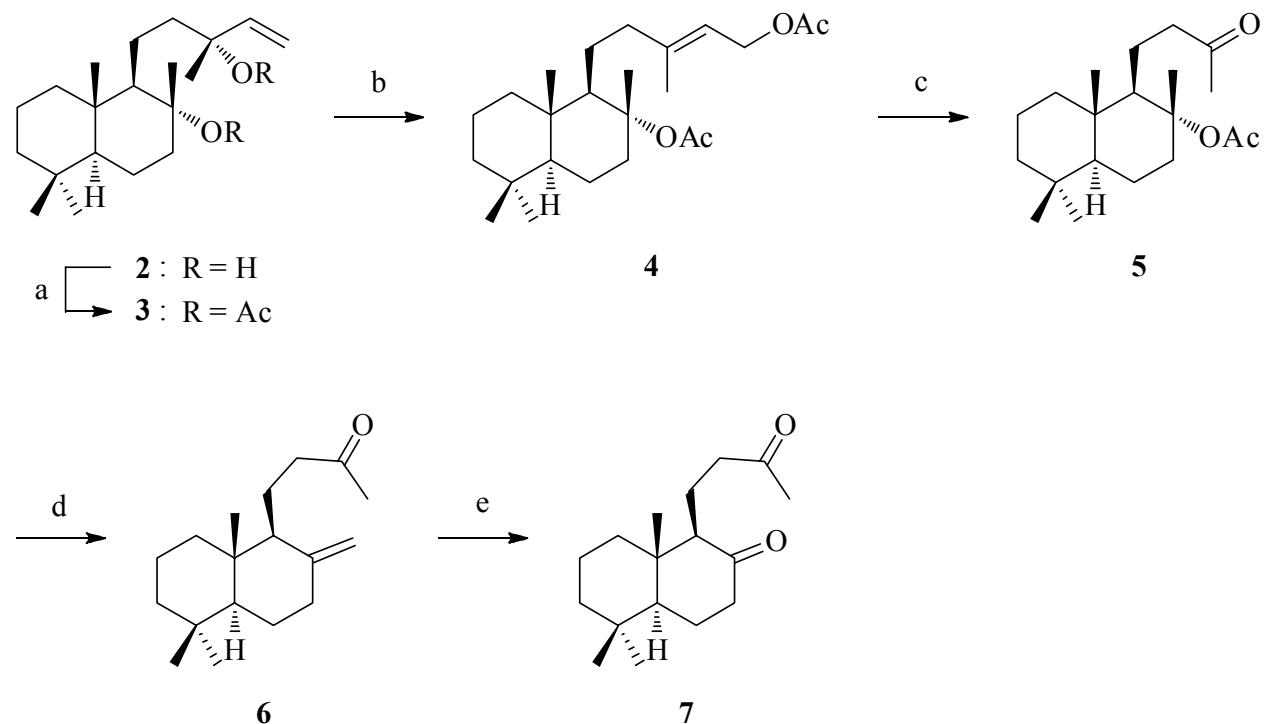

Scheme 1. Synthesis of diketone 7 from (-)-sclareol (2). Reagents and conditions: (a) AcCl, $\mathrm{PhNMe}_{2}, \mathrm{CH}_{2} \mathrm{Cl}_{2}$, RT, 26 h, 96\%; (b) 0.94 mol\% $\mathrm{PdCl}_{2}(\mathrm{MeCN})_{2}$, THF, RT, 6 h, 100\%; (c) $\mathrm{O}_{3}$, $\mathrm{CH}_{2} \mathrm{Cl}_{2},-70^{\circ} \mathrm{C}, 1 \mathrm{~h}$, then $\mathrm{Me}_{2} \mathrm{~S},-70^{\circ} \mathrm{C} \rightarrow \mathrm{RT}, 66 \%$; (d) $\mathrm{NaHCO}_{3}, \mathrm{DMSO} 150^{\circ} \mathrm{C}, 6 \mathrm{~h}, 93 \%$ (88:12 mixture of isomers); (e) $\mathrm{O}_{3}, \mathrm{CH}_{2} \mathrm{Cl}_{2},-78^{\circ} \mathrm{C}, 35 \mathrm{~min}$, then $\mathrm{Ph}_{3} \mathrm{P},-78^{\circ} \mathrm{C} \rightarrow \mathrm{RT}, 63 \%$. 
The route from (-)-sclareol (2) to keto olefin $6^{18}$ followed the protocol of Zahra and Waegell with slight modifications. ${ }^{19}$ To this end, $\mathbf{2}$ was converted to its diacetate $\mathbf{3}$ that underwent a clean $\mathrm{Pd}(\mathrm{II})$-catalyzed rearrangement to give the isomeric diacetate $\mathbf{4}$. Ozonolysis of $\mathbf{4}$ followed by regioselective elimination of acetic acid delivered the desired exocyclic olefin $\mathbf{6}$ along with a minor double bond isomer. Subsequent ozonolysis of this mixture containing $\mathbf{6}$ as the major component $^{20}$ gave rise to the pure diketone 7 in five steps and $37 \%$ overall yield from 2 .

An alternative route to diketone 7 from sclareol diacetate $\mathbf{3}$ is illustrated in Scheme 2. Chemoselective hydrolysis of $\mathbf{3}$ to furnish monoacetate $\mathbf{8}^{19,21}$ set the stage for a regioselective elimination to yield (+)-manool (9) as the major product. Ozonolysis of this mixture containing 9 as the major component in the presence of sodium hydroxide ${ }^{17 \mathrm{c}}$ afforded diketone 7 in four steps and $25 \%$ overall yield from 2 . As anticipated, analogous treatment of (+)-13-epi-manool (10) led to diketone 7 as well.

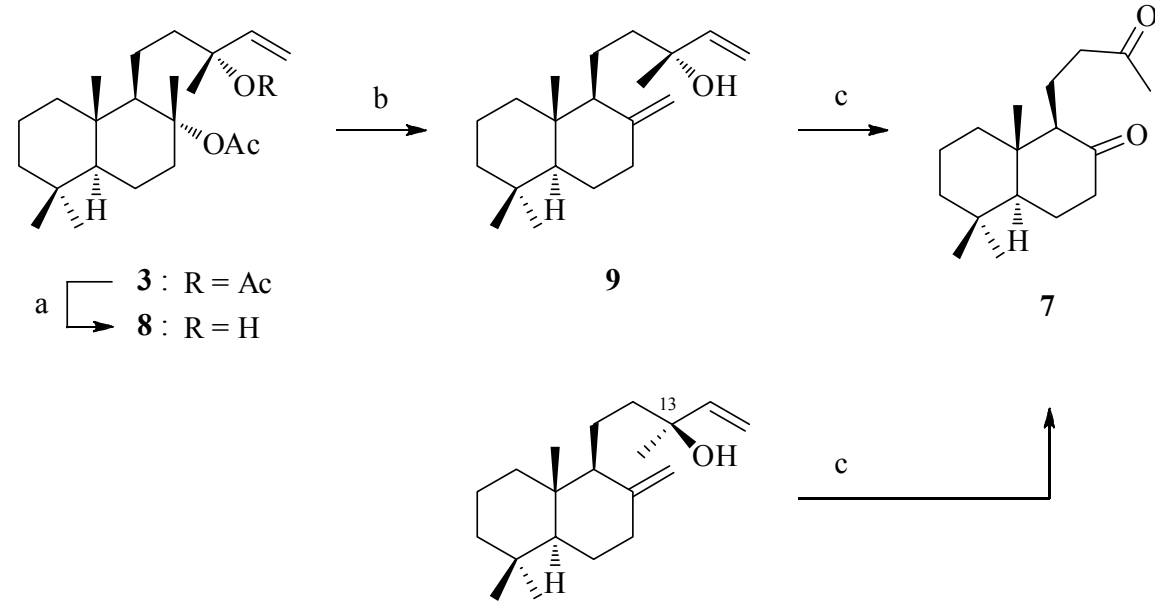

10

Scheme 2. Alternative syntheses of diketone 7 from (-)-sclareol via (+)-manool (9) and from (+)-13-epi-manool (10). Reagents and conditions: (a) $\mathrm{KOH}, \mathrm{H}_{2} \mathrm{O}, \mathrm{EtOH}, \mathrm{RT}, 48 \mathrm{~h}, 60 \%$; (b) $\mathrm{NaHCO}_{3}, \mathrm{DMSO}, 150^{\circ} \mathrm{C}, 6 \mathrm{~h}, 90 \%$ (mixture of isomers with $77 \%$ major product 9); (c) $\mathrm{O}_{3}$, toluene, $10 \%$ aq. $\mathrm{NaOH},-10^{\circ} \mathrm{C}, 35 \mathrm{~min}, 48 \%$.

Completion of the synthesis of (+)-totarol (1) from diketone 7 is shown in Scheme 3. Gratifyingly, the intramolecular aldol condensation of $7^{17 \mathrm{e}-\mathrm{h}}$ could be readily coupled in a onepot fashion with an enone $\alpha$-alkylation ${ }^{15,22}$ using isopropyl iodide to directly give the desired enone $\mathbf{1 1}^{8,12}$ as the major product. Finally, $\mathrm{Cu}(\mathrm{II})$-mediated oxidative aromatization ${ }^{12,23}$ of this mixture cleanly led to the pure diterpene $\mathbf{1}$ in high yield identical to the natural product in all respects.

In conclusion, short synthetic routes to enantiopure (+)-totarol (1) have been developed. Since (-)-sclareol (2) can be obtained by extraction of the plant Salvia sclarea, it is currently the 
preferred source out of the three diterpenes $\mathbf{2}, \mathbf{9}$, and $\mathbf{1 0}$ for the semisynthesis of $\mathbf{1}$ according to reaction sequences depicted in Schemes 1-3.

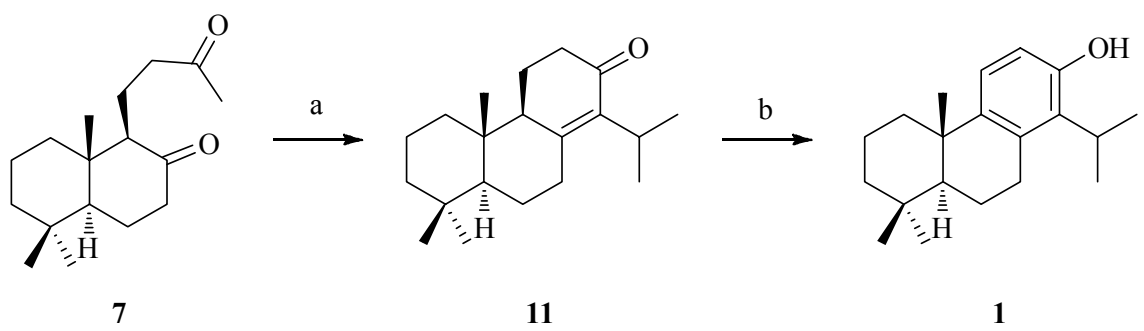

Scheme 3. Streamlined conversion of diketone 7 to (+)-totarol (1). Reagents and conditions: (a) $t$-BuOK, $t$-BuOH, reflux, $50 \mathrm{~min}$, then $i$-PrI, reflux, $1 \mathrm{~h}, 56 \%$ (9:1.5:3.5:86 mixture of isomers containing 11 as the major product); (b) $\mathrm{CuBr}_{2}, \mathrm{LiBr}, \mathrm{MeCN}$, reflux, $20 \mathrm{~h}, 91 \%$.

\section{Experimental Section}

General. All reactions requiring exclusion of moisture were run under argon using heat gundried glassware. Solvents were dried by distillation from $\mathrm{Na} / \mathrm{K}$ and benzophenone (THF), $\mathrm{Na}$ (toluene), $\mathrm{CaH}_{2}\left(\mathrm{Et}_{2} \mathrm{O}\right)$, or by passing through activated alumina $\left(\mathrm{CH}_{2} \mathrm{Cl}_{2}\right)$. All commercially available compounds were used as received unless stated otherwise. Flash chromatography: Merck silica gel $60(40-63 \mu \mathrm{m})$. Thin layer chromatography: Merck silica gel $60 \mathrm{~F}_{254}$ plates; for visualization of the spots a solution of anisaldehyde $(6 \mathrm{~g})$ and sulfuric acid $(2.5 \mathrm{~mL})$ in EtOH $(250 \mathrm{~mL})$ was used. Melting points: Kleinfeld Labortechnik Electrothermal IA 9100 apparatus. Optical rotation: Perkin-Elmer 341 polarimeter. ${ }^{1} \mathrm{H}$ and ${ }^{13} \mathrm{C}$ NMR: Bruker DRX-500 $\left({ }^{1} \mathrm{H}: 500\right.$ $\mathrm{MHz},{ }^{13} \mathrm{C}: 126 \mathrm{MHz}, \mathrm{CDCl}_{3}$, calibrated to the residual resonance of the solvent) or Bruker AC$300\left({ }^{1} \mathrm{H}: 300 \mathrm{MHz},{ }^{13} \mathrm{C}: 75.4 \mathrm{MHz}, \mathrm{CDCl}_{3}\right.$, calibrated to the residual resonance of the solvent). Mass spectra: Hewlett Packard 5890 GC coupled with a Hewlett Packard 5972 detector and Agilent 6890N GC coupled with an Agilent 5973N detector (GC/MS).

Sclareol diacetate (3). (-)-Sclareol (2) (50.0 g, $0.162 \mathrm{~mol})$ was stirred with $N, N$-dimethylaniline $(98.0 \mathrm{~g}, 0.809 \mathrm{~mol})$ and dichloromethane $(60 \mathrm{~mL})$ in a $500 \mathrm{~mL}$ flask for $30 \mathrm{~min}$ at RT. Acetyl chloride $(42.0 \mathrm{~g}, 0.531 \mathrm{~mol})$ dissolved in dichloromethane $(30 \mathrm{~mL})$ was added dropwise over a period of $1 \mathrm{~h}$. After complete addition, the mixture was stirred for $26 \mathrm{~h}$ at RT. Subsequently, diethyl ether $(500 \mathrm{~mL})$, ice $(250 \mathrm{~g})$, and $2 \mathrm{~N} \mathrm{HCl}(300 \mathrm{~mL})$ were added. The layers were separated, and the organic layer was washed with $2 \mathrm{~N} \mathrm{HCl}(400 \mathrm{~mL})$, brine $(100 \mathrm{~mL})$, saturated $\mathrm{Na}_{2} \mathrm{CO}_{3}$ solution $(100 \mathrm{~mL})$, and then again with brine $(100 \mathrm{~mL})$ before drying with $\mathrm{MgSO}_{4}$ and concentration in vacuum. The crude material was filtered through a pad of silica gel using ethyl acetate as eluent to give $3(61.310 \mathrm{~g}, 96 \%)$ as pale yellow solid; $R_{\mathrm{f}} 0.55$ (pentane/EtOAc $\left.5: 1\right) ;{ }^{1} \mathrm{H}$ NMR $(300 \mathrm{MHz}) \delta 0.71\left(\mathrm{~s}, 3 \mathrm{H}, \mathrm{CH}_{3}\right), 0.75\left(\mathrm{~s}, 3 \mathrm{H}, \mathrm{CH}_{3}\right), 0.79\left(\mathrm{~s}, 3 \mathrm{H}, \mathrm{CH}_{3}\right), 0.90(\mathrm{dd}, J=2.1$, 
$12.3 \mathrm{~Hz}, 2 \mathrm{H}), 1.00-1.40$ (m, 7H), 1.38 (s, 3H, $\left.\mathrm{CH}_{3}\right), 1.47$ (s, 3H, $\left.\mathrm{CH}_{3}\right), 1.50-1.70$ (m, 4H), 1.80$1.88(\mathrm{~m}, 2 \mathrm{H}), 1.87\left(\mathrm{~s}, 3 \mathrm{H}, \mathrm{CH}_{3}\right), 1.94\left(\mathrm{~s}, 3 \mathrm{H}, \mathrm{CH}_{3}\right), 2.50-2.60(\mathrm{~m}, 1 \mathrm{H}), 5.05(\mathrm{dd}, J=0.9,11.0$ $\mathrm{Hz}, 1 \mathrm{H}), 5.08(\mathrm{dd}, J=0.9,17.5 \mathrm{~Hz}, 1 \mathrm{H}), 5.89(\mathrm{dd}, J=11.0,17.5 \mathrm{~Hz}, 1 \mathrm{H}) ;{ }^{13} \mathrm{C} \mathrm{NMR}(75.4 \mathrm{MHz})$ $\delta 15.73\left(\mathrm{CH}_{3}\right), 18.31\left(\mathrm{CH}_{2}\right), 19.46\left(\mathrm{CH}_{2}\right), 19.94\left(\mathrm{CH}_{2}\right), 20.42\left(\mathrm{CH}_{3}\right), 21.38\left(\mathrm{CH}_{3}\right), 22.19\left(\mathrm{CH}_{3}\right)$, $22.87\left(\mathrm{CH}_{3}\right), 23.54\left(\mathrm{CH}_{3}\right), 33.08\left(\mathrm{CH}_{3}\right), 33.29(\mathrm{C}), 38.75\left(\mathrm{CH}_{2}\right), 39.43\left(\mathrm{CH}_{2}\right), 39.48(\mathrm{C}), 41.87$ $\left(\mathrm{CH}_{2}\right), 42.58\left(\mathrm{CH}_{2}\right), 55.62(\mathrm{CH}), 58.68(\mathrm{CH}), 83.19(\mathrm{C}), 87.94(\mathrm{C}), 113.06\left(\mathrm{CH}_{2}\right), 141.92(\mathrm{CH})$, 169.82 (C), 170.23 (C); MS (GC/MS, $70 \mathrm{eV}) \mathrm{m} / \mathrm{z}$ (rel. intensity): 272 [M+ -2 HOAc] (20), 257 (50), 244 (5), 229 (9), 217 (12), 201 (14), 189 (19), 177 (26), 161 (23), 147 (20), 137 (100), 121 (45).

Diacetate (4). A suspension of $\mathrm{PdCl}_{2}(50 \mathrm{mg}, 0.282 \mathrm{mmol})$ in acetonitrile $(1 \mathrm{~mL})$ was stirred under argon atmosphere for $5 \mathrm{~d}$ at RT. Then the yellow suspension was added to a solution of sclareol diacetate (3) (11.765 g, $30 \mathrm{mmol})$ in dry THF $(300 \mathrm{~mL})$. The mixture was stirred for $6 \mathrm{~h}$ at RT and then concentrated in vacuum. The resultant crude product was suspended in pentane $(40 \mathrm{~mL})$ and purified by filtration over silica gel (column diameter $3 \mathrm{~cm}$, height $15 \mathrm{~cm}$ ) using diethyl ether $(800 \mathrm{~mL})$ as eluent. After concentrating under reduced pressure, diacetate $4(11.725$ $\mathrm{g}, 100 \%$ ) was obtained as pale yellow oil; $R_{\mathrm{f}} 0.49$ (pentane/EtOAc 5:1); ${ }^{1} \mathrm{H}$ NMR (300 MHz) $\delta$ $0.71\left(\mathrm{~s}, 3 \mathrm{H}, \mathrm{CH}_{3}\right), 0.76\left(\mathrm{~s}, 3 \mathrm{H}, \mathrm{CH}_{3}\right), 0.80\left(\mathrm{~s}, 3 \mathrm{H}, \mathrm{CH}_{3}\right), 0.92(\mathrm{~m}, 2 \mathrm{H}), 1.00-1.72(\mathrm{~m}, 9 \mathrm{H}), 1.39$ $\left(\mathrm{s}, 3 \mathrm{H}, \mathrm{CH}_{3}\right), 1.65\left(\mathrm{~s}, 3 \mathrm{H}, \mathrm{CH}_{3}\right), 1.91-2.10(\mathrm{~m}, 4 \mathrm{H}), 1.87\left(\mathrm{~s}, 3 \mathrm{H}, \mathrm{CH}_{3}\right), 1.99\left(\mathrm{~s}, 3 \mathrm{H}, \mathrm{CH}_{3}\right)$, 2.50-2.60 (m, 1H), $4.51(\mathrm{~d}, J=7.1 \mathrm{~Hz}, 2 \mathrm{H}), 5.26$ (dd, $J=6.4,7.1 \mathrm{~Hz}, 1 \mathrm{H})$.

Ketone (5). To a $500 \mathrm{~mL}$ three-necked flask a solution of diacetate 4 (12.800 g, $32.6 \mathrm{mmol})$ in dry $\mathrm{CH}_{2} \mathrm{Cl}_{2}(250 \mathrm{~mL})$ was added. This solution was cooled to $-70^{\circ} \mathrm{C}$ and ozonized at this temperature for $1 \mathrm{~h}$ when a constant blue coloration was reached. To remove excess ozone, the reaction mixture was purged with $\mathrm{N}_{2}$. Then dimethyl sulfide (12.8 g) was added, and the mixture was allowed to warm to RT overnight with stirring. After concentration under reduced pressure, the crude yellow product (16.604 g) was purified by column chromatography over silica gel (column diameter $5 \mathrm{~cm}$, height $50 \mathrm{~cm}$ ) using pentane/ethyl acetate $5: 1$ as eluent to give ketone 5 $(6.890 \mathrm{~g}, 66 \%)$ as a white solid; $R_{\mathrm{f}} 0.30$ (pentane/EtOAc 5:1); ${ }^{1} \mathrm{H}$ NMR $(300 \mathrm{MHz}) \delta 0.76(\mathrm{~s}, 3 \mathrm{H}$, $\left.\mathrm{CH}_{3}\right), 0.82\left(\mathrm{~s}, 3 \mathrm{H}, \mathrm{CH}_{3}\right), 0.85\left(\mathrm{~s}, 3 \mathrm{H}, \mathrm{CH}_{3}\right), 0.86-1.00(\mathrm{~m}, 2 \mathrm{H}), 1.08-1.43(\mathrm{~m}, 4 \mathrm{H}), 1.45(\mathrm{~s}, 3 \mathrm{H}$, $\left.\mathrm{CH}_{3}\right), 1.48-1.77(\mathrm{~m}, 7 \mathrm{H}), 1.91\left(\mathrm{~s}, 3 \mathrm{H}, \mathrm{CH}_{3}\right), 2.12\left(\mathrm{~s}, 3 \mathrm{H}, \mathrm{CH}_{3}\right), 2.45-2.66(\mathrm{~m}, 3 \mathrm{H}) ;{ }^{13} \mathrm{C}$ NMR (75.4 MHz) $\delta 15.51\left(\mathrm{CH}_{3}\right), 18.21\left(\mathrm{CH}_{2}\right), 19.59\left(\mathrm{CH}_{2}\right), 19.91\left(\mathrm{CH}_{2}\right), 20.39\left(\mathrm{CH}_{3}\right), 21.38\left(\mathrm{CH}_{3}\right)$, $22.95\left(\mathrm{CH}_{3}\right), 29.85\left(\mathrm{CH}_{3}\right), 33.10(\mathrm{C}), 33.29\left(\mathrm{CH}_{3}\right), 38.75\left(\mathrm{CH}_{2}\right), 39.46(\mathrm{C}), 39.61\left(\mathrm{CH}_{2}\right), 41.80$ $\left(\mathrm{CH}_{2}\right), 46.64\left(\mathrm{CH}_{2}\right), 55.60(\mathrm{CH}), 58.04(\mathrm{CH}), 88.03(\mathrm{C}), 170.04(\mathrm{C}), 209.21(\mathrm{C})$.

Keto olefin (6). In a $100 \mathrm{~mL}$ flask ketone 5 (6.890 g, $21.36 \mathrm{mmol})$ was suspended in DMSO (40 $\mathrm{mL})$, and $\mathrm{NaHCO}_{3}(2.690 \mathrm{~g}, 32 \mathrm{mmol})$ was added. This mixture was stirred under argon atmosphere at $150^{\circ} \mathrm{C}$ for $6 \mathrm{~h}$. After cooling the mixture to RT, water (200 mL) was added, and the resulting suspension was extracted with $\mathrm{Et}_{2} \mathrm{O}(2 \times 200 \mathrm{~mL})$. The organic layer was separated, washed with water $(200 \mathrm{~mL})$ and brine $(50 \mathrm{~mL})$, dried with $\mathrm{MgSO}_{4}$ and concentrated under reduced pressure. The crude product $(5.430 \mathrm{~g}$, yellow oil) was purified by column chromatography over silica gel using pentane/EtOAc 10:1 as eluent to give keto olefin 6 (5.233 $\mathrm{g}, 93 \%)$ as a pale yellow oil (88:12 mixture of isomers with 6 as the major component); $R_{\mathrm{f}} 0.57$ 
(pentane/EtOAc 5:1); ${ }^{1} \mathrm{H}$ NMR (300 MHz) $\delta 0.62\left(\mathrm{~s}, 3 \mathrm{H}, \mathrm{CH}_{3}\right), 0.73\left(\mathrm{~s}, 3 \mathrm{H}, \mathrm{CH}_{3}\right), 0.79(\mathrm{~s}, 3 \mathrm{H}$, $\left.\mathrm{CH}_{3}\right), 0.76-1.55(\mathrm{~m}, 10 \mathrm{H}), 1.61-1.92(\mathrm{~m}, 3 \mathrm{H}), 2.03\left(\mathrm{~s}, 3 \mathrm{H}, \mathrm{CH}_{3}\right), 2.18-2.35(\mathrm{~m}, 2 \mathrm{H}), 2.45-2.56$ $(\mathrm{m}, 1 \mathrm{H}), 4.36(\mathrm{~s}, 1 \mathrm{H}), 4.75(\mathrm{~d}, J=1.0 \mathrm{~Hz}, 1 \mathrm{H}) ;{ }^{13} \mathrm{C} \mathrm{NMR}(75.4 \mathrm{MHz}) \delta 14.29\left(\mathrm{CH}_{3}\right), 17.51$ $\left(\mathrm{CH}_{2}\right), 19.34\left(\mathrm{CH}_{2}\right), 21.69\left(\mathrm{CH}_{3}\right), 24.43\left(\mathrm{CH}_{2}\right), 30.02\left(\mathrm{CH}_{3}\right), 33.56(\mathrm{C}), 33.61\left(\mathrm{CH}_{3}\right), 38.26$ $\left(\mathrm{CH}_{2}\right), 38.96\left(\mathrm{CH}_{2}\right), 39.76(\mathrm{C}), 42.11\left(\mathrm{CH}_{2}\right), 42.91\left(\mathrm{CH}_{2}\right), 55.48(\mathrm{CH}), 56.26(\mathrm{CH}), 106.29$ $\left(\mathrm{CH}_{2}\right), 148.35(\mathrm{C}), 209.51(\mathrm{C})$; MS (GC/MS, $\left.70 \mathrm{eV}\right) \mathrm{m} / \mathrm{z}$ (rel. intensity): $262\left[\mathrm{M}^{+}\right]$(20), 247 (17), 244 (26), 229 (39), 204 (46), 191 (26), 189 (26), 177 (38), 159 (27), 147 (20), 137 (100), 121 (26), 107 (60), 95 (80).

Diketone (7) from keto olefin (6). Keto olefin 6 (5.100 g, $19.43 \mathrm{mmol})$ prepared as described above was dissolved in dry $\mathrm{CH}_{2} \mathrm{Cl}_{2}(120 \mathrm{~mL})$ in a $500 \mathrm{~mL}$ two-necked flask with a gas inlet tube. This solution was cooled to $-78^{\circ} \mathrm{C}$ and ozonized at this temperature with vigorous stirring for 35 min when a constant blue coloration was reached. The ozone generator was turned off, and the reaction mixture was further stirred for $30 \mathrm{~min}$ at $-78^{\circ} \mathrm{C}$. To remove excess ozone, the reaction mixture was purged with $\mathrm{N}_{2}$ for $10 \mathrm{~min}$. Then triphenylphosphine $(6.150 \mathrm{~g})$ was added, and the suspension was stirred for additional $2 \mathrm{~h}$ at $-78^{\circ} \mathrm{C}$. The cooling bath was removed, and the mixture was allowed to warm to RT overnight with stirring. After removal of the solvent in vacuum, the crude yellow product was purified by column chromatography over silica gel using pentane/EtOAc 10:1 $\rightarrow$ 5:1 as eluent to give diketone $7(3.230 \mathrm{~g}, 63 \%)$ as a colorless oil; $R_{\mathrm{f}} 0.19$ (pentane/EtOAc 5:1); ${ }^{1} \mathrm{H}$ NMR $(300 \mathrm{MHz}) \delta 0.75\left(\mathrm{~s}, 3 \mathrm{H}, \mathrm{CH}_{3}\right), 0.87\left(\mathrm{~s}, 3 \mathrm{H}, \mathrm{CH}_{3}\right), 0.98(\mathrm{~s}, 3 \mathrm{H}$, $\left.\mathrm{CH}_{3}\right), 1.17-1.33(\mathrm{~m}, 2 \mathrm{H}), 1.42-1.70(\mathrm{~m}, 6 \mathrm{H}), 1.76-1.88(\mathrm{~m}, 2 \mathrm{H}), 2.03-2.14(\mathrm{~m}, 2 \mathrm{H}), 2.12$ (s, $\left.3 \mathrm{H}, \mathrm{CH}_{3}\right), 2.15-2.35(\mathrm{~m}, 2 \mathrm{H}), 2.39-2.48(\mathrm{~m}, 1 \mathrm{H}), 2.57-2.69(\mathrm{~m}, 1 \mathrm{H}) ;{ }^{13} \mathrm{C} \mathrm{NMR}(75.4 \mathrm{MHz}) \delta$ $14.55\left(\mathrm{CH}_{3}\right), 16.11\left(\mathrm{CH}_{2}\right), 18.96\left(\mathrm{CH}_{2}\right), 21.66\left(\mathrm{CH}_{3}\right), 23.95\left(\mathrm{CH}_{2}\right), 29.89\left(\mathrm{CH}_{3}\right), 33.50(\mathrm{C}), 33.67$ $\left(\mathrm{CH}_{3}\right), 39.10\left(\mathrm{CH}_{2}\right), 41.84\left(\mathrm{CH}_{2}\right), 42.57\left(\mathrm{CH}_{2}\right), 42.68(\mathrm{C}), 42.76\left(\mathrm{CH}_{2}\right), 54.12(\mathrm{CH}), 63.14(\mathrm{CH})$, 209.22 (C), 212.34 (C); MS (GC/MS, 70 eV) m/z (rel. intensity): $264\left[\mathrm{M}^{+}\right]$(10), 249 (100), 231 (15), 179 (13), 173 (11), 137 (16), 121 (20), 109 (15), 95 (23).

Sclareol monoacetate (8). Sclareol diacetate 3 (61.315 g, $0.156 \mathrm{~mol})$ was dissolved in 99\% $\mathrm{EtOH}(530 \mathrm{~mL})$ in a $1 \mathrm{~L}$ flask cooled with ice water to $15^{\circ} \mathrm{C}$, and a solution of $\mathrm{KOH}(8.44 \mathrm{~g}$, $0.150 \mathrm{~mol})$ in $\mathrm{H}_{2} \mathrm{O} / \mathrm{EtOH}(50 \%, 70 \mathrm{~mL})$ was added dropwise over $15 \mathrm{~min}$ to the stirred mixture. The orange reaction mixture was stirred for $24 \mathrm{~h}$ at RT. Subsequently, KOH (1.6 g, $0.027 \mathrm{~mol}$ ) was added, the suspension was stirred for additional $24 \mathrm{~h}$ and then concentrated in vacuum at $40^{\circ} \mathrm{C}$ bath temperature. Water $(300 \mathrm{~mL})$ was added, and the resulting suspension was extracted with EtOAc $(2 \times 500 \mathrm{~mL})$. The combined organic extracts were dried with $\mathrm{MgSO}_{4}$ and filtered through a pad of silica gel. After removal of the solvent in vacuum, the crude white product was recrystallized from a 2:1 EtOAc/hexane mixture $(90 \mathrm{~mL})$ to give monoacetate $8(32.57 \mathrm{~g}, 60 \%)$ as a white solid (the filtrate containing sclareol, sclareol monoacetate and sclareol diacetate could be used again); $R_{\mathrm{f}} 0.20$ (pentane/EtOAc 5:1); ${ }^{1} \mathrm{H}$ NMR $(300 \mathrm{MHz}) \delta 0.71\left(\mathrm{~s}, 3 \mathrm{H}, \mathrm{CH}_{3}\right), 0.76(\mathrm{~s}$, $\left.3 \mathrm{H}, \mathrm{CH}_{3}\right), 0.79\left(\mathrm{~s}, 3 \mathrm{H}, \mathrm{CH}_{3}\right), 0.90(\mathrm{dd}, J=1.9,12.3 \mathrm{~Hz}, 2 \mathrm{H}), 1.02-1.50(\mathrm{~m}, 8 \mathrm{H}), 1.22(\mathrm{~s}, 3 \mathrm{H}$, $\left.\mathrm{CH}_{3}\right), 1.37\left(\mathrm{~s}, 3 \mathrm{H}, \mathrm{CH}_{3}\right), 1.53-1.70(\mathrm{~m}, 7 \mathrm{H}), 1.86\left(\mathrm{~s}, 3 \mathrm{H}, \mathrm{CH}_{3}\right), 2.53-2.59(\mathrm{~m}, 1 \mathrm{H}), 5.00(\mathrm{dd}, J=$ $1.2,10.7 \mathrm{~Hz}, 1 \mathrm{H}), 5.14(\mathrm{dd}, J=1.3,17.4 \mathrm{~Hz}, 1 \mathrm{H}), 5.83(\mathrm{dd}, J=10.7,17.4 \mathrm{~Hz}, 1 \mathrm{H}) ;{ }^{13} \mathrm{C}$ NMR (75.4 MHz) $\delta 15.71\left(\mathrm{CH}_{3}\right), 18.29\left(\mathrm{CH}_{2}\right), 19.83\left(\mathrm{CH}_{2}\right), 19.96\left(\mathrm{CH}_{2}\right), 20.42\left(\mathrm{CH}_{3}\right), 21.40\left(\mathrm{CH}_{3}\right)$, 
$22.95\left(\mathrm{CH}_{3}\right), 27.86\left(\mathrm{CH}_{3}\right), 33.09(\mathrm{C}), 33.30\left(\mathrm{CH}_{3}\right), 38.77\left(\mathrm{CH}_{2}\right), 39.50(\mathrm{C}), 39.62\left(\mathrm{CH}_{2}\right), 41.89$ $\left(\mathrm{CH}_{2}\right), 45.33\left(\mathrm{CH}_{2}\right), 55.63(\mathrm{CH}), 58.86(\mathrm{CH}), 73.55(\mathrm{C}), 88.28(\mathrm{C}), 111.76\left(\mathrm{CH}_{2}\right), 145.00(\mathrm{CH})$, $170.13(\mathrm{C})$.

(+)-Manool (9). In a $500 \mathrm{~mL}$ flask sclareol monoacetate 8 (32.253 g, $92.00 \mathrm{mmol})$ was dissolved in DMSO $(220 \mathrm{~mL})$, and powdered $\mathrm{NaHCO}_{3}(11.592 \mathrm{~g}, 138 \mathrm{mmol})$ was added. This suspension was stirred under argon atmosphere at $150^{\circ} \mathrm{C}$ for $6 \mathrm{~h}$. After cooling to RT, water $(400 \mathrm{~mL})$ was added, and the resulting suspension was extracted with $\mathrm{Et}_{2} \mathrm{O}(2 \times 400 \mathrm{~mL})$. The combined extracts were washed with water $(500 \mathrm{~mL})$ and brine $(100 \mathrm{~mL})$, dried with $\mathrm{MgSO}_{4}$ and concentrated in vacuum. The residue was purified by column chromatography over silica gel using pentane/EtOAc 10:1 $\rightarrow$ 5:1 as eluent to give (+)-manool (9) $(24.181 \mathrm{~g}, 90 \%)$ as a colorless oil (mixture of isomers with a manool content of $77 \%$ according to GC); $R_{\mathrm{f}} 0.50$ (pentane/EtOAc 5:1); ${ }^{1} \mathrm{H}$ NMR (300 MHz) $\delta 0.60\left(\mathrm{~s}, 3 \mathrm{H}, \mathrm{CH}_{3}\right), 0.72\left(\mathrm{~s}, 3 \mathrm{H}, \mathrm{CH}_{3}\right), 0.80\left(\mathrm{~s}, 3 \mathrm{H}, \mathrm{CH}_{3}\right), 0.94-1.90$ (m, $16 \mathrm{H}), 1.20\left(\mathrm{~s}, 3 \mathrm{H}, \mathrm{CH}_{3}\right), 2.25-2.35(\mathrm{~m}, 1 \mathrm{H}), 4.40(\mathrm{~d}, J=1.1 \mathrm{~Hz}, 1 \mathrm{H}), 4.73(\mathrm{~d}, J=1.1 \mathrm{~Hz}$, $1 \mathrm{H}), 5.00(\mathrm{dd}, J=1.2,10.7 \mathrm{~Hz}, 1 \mathrm{H}), 5.14(\mathrm{dd}, J=1.2,17.4 \mathrm{~Hz}, 1 \mathrm{H}), 5.83(\mathrm{dd}, J=10.7,17.4 \mathrm{~Hz}$, $1 \mathrm{H}) ;{ }^{13} \mathrm{C}$ NMR $(75.4 \mathrm{MHz}) \delta 14.43\left(\mathrm{CH}_{3}\right), 17.64\left(\mathrm{CH}_{2}\right), 19.36\left(\mathrm{CH}_{2}\right), 21.70\left(\mathrm{CH}_{3}\right), 24.43\left(\mathrm{CH}_{2}\right)$, $28.01\left(\mathrm{CH}_{3}\right), 33.55(\mathrm{C}), 33.61\left(\mathrm{CH}_{3}\right), 38.34\left(\mathrm{CH}_{2}\right), 39.06\left(\mathrm{CH}_{2}\right), 39.84(\mathrm{C}), 41.34\left(\mathrm{CH}_{2}\right), 42.17$ $\left(\mathrm{CH}_{2}\right), 55.55(\mathrm{CH}), 57.21(\mathrm{CH}), 73.65(\mathrm{C}), 106.31\left(\mathrm{CH}_{2}\right), 111.59\left(\mathrm{CH}_{2}\right), 145.13(\mathrm{CH}), 148.74$ (C); MS (GC/MS, $70 \mathrm{eV}) \mathrm{m} / z$ (rel. intensity): $290\left[\mathrm{M}^{+}\right]$(2), 272 (14), 257 (60), 244 (10), 229 (9), 215 (5), 204 (23), 189 (44), 177 (26), 161 (24), 149 (23), 137 (100), 121 (53).

Diketone (7) from (+)-manool (9). (+)-Manool (9) (2.720 g, $9.60 \mathrm{mmol})$ prepared as described above was dissolved in toluene $(37 \mathrm{~mL})$ in a $250 \mathrm{~mL}$ two-necked flask with a gas inlet tube. After addition of $10 \%$ aqueous $\mathrm{NaOH}$ solution $(20 \mathrm{~mL})$, the mixture was cooled to $-10^{\circ} \mathrm{C}$ and ozonized at this temperature for $35 \mathrm{~min}$ with vigorous stirring. The ozone generator was turned off, the reaction mixture was purged with $\mathrm{N}_{2}$ for 10 min and then warmed to RT. The layers were separated (the water layer has a yellow coloration), and the colorless organic layer was washed with $2 \mathrm{~N} \mathrm{NaOH}(20 \mathrm{~mL})$, dried with $\mathrm{MgSO}_{4}$ and concentrated in vacuum. The residue was purified by column chromatography over silica gel using pentane/EtOAc 10:1 $\rightarrow$ 5:1 as eluent to give $7(1.180 \mathrm{~g}, 48 \%)$ as a colorless oil.

Cyclohexenone (11). Potassium-t-butoxide (7.605 g, $67.77 \mathrm{mmol})$ was suspended in $t$ - $\mathrm{BuOH}$ (30 $\mathrm{mL}$ ) in a $500 \mathrm{~mL}$ three-necked flask under argon atmosphere and heated to reflux. To this suspension a solution of diketone $7(6.45 \mathrm{~g}, 24.39 \mathrm{mmol})$ in $t$ - $\mathrm{BuOH}(70 \mathrm{~mL})$ was added over 3 $\mathrm{min}$. The mixture was heated at reflux for $50 \mathrm{~min}$. Subsequently, a solution of isopropyl iodide $(3.850 \mathrm{~g}, 22.65 \mathrm{mmol})$ in $t$ - $\mathrm{BuOH}(200 \mathrm{~mL})$ was added to the refluxing mixture over $1 \mathrm{~h}$, and the resultant mixture was refluxed for another $1 \mathrm{~h}$. After cooling to $\mathrm{RT} \mathrm{Et}_{2} \mathrm{O}(400 \mathrm{~mL})$ was added, and the organic layer was washed two times with water, dried with $\mathrm{MgSO}_{4}$ and concentrated in vacuum. The crude product was purified by column chromatography over silica gel using pentane/EtOAc 20:1 as eluent to give cyclohexenone 11 (3.949 g, 56\%) as a 9:1.5:3.5:86 (GC/MS) mixture of four isomers with a molar mass of 288 as an orange oil. Data of the major product 11: $R_{\mathrm{f}} 0.49$ (pentane/EtOAc 10:1); ${ }^{1} \mathrm{H}$ NMR (300 MHz) $\delta 0.76\left(\mathrm{~s}, 3 \mathrm{H}, \mathrm{CH}_{3}\right), 0.88-1.28$ (m, 4H), 0.89 (s, 3H, $\left.\mathrm{CH}_{3}\right), 0.95$ (s, 3H, $\left.\mathrm{CH}_{3}\right), 1.15$ (d, $\left.J=7.1 \mathrm{~Hz}, 3 \mathrm{H}, \mathrm{CH}_{3}\right), 1.21(\mathrm{~d}, J=7.1 \mathrm{~Hz}$, 
$\left.3 \mathrm{H}, \mathrm{CH}_{3}\right), 1.42-1.73(\mathrm{~m}, 5 \mathrm{H}), 1.78-1.97(\mathrm{~m}, 3 \mathrm{H}), 2.06-2.40(\mathrm{~m}, 3 \mathrm{H}), 3.10-3.23(\mathrm{~m}, 2 \mathrm{H}) ;{ }^{13} \mathrm{C}$ NMR $(75.4 \mathrm{MHz}) \delta 14.61\left(\mathrm{CH}_{3}\right), 18.84\left(\mathrm{CH}_{2}\right), 19.67\left(\mathrm{CH}_{2}\right), 20.43\left(\mathrm{CH}_{3}\right), 21.82\left(\mathrm{CH}_{3}\right), 21.98$ $\left(\mathrm{CH}_{3}\right), 22.45\left(\mathrm{CH}_{2}\right), 26.60\left(\mathrm{CH}_{3}\right), 31.39\left(\mathrm{CH}_{2}\right), 33.34(\mathrm{C}), 33.53(\mathrm{CH}), 37.92\left(\mathrm{CH}_{2}\right), 38.72\left(\mathrm{CH}_{2}\right)$, $39.93(\mathrm{C}), 41.80\left(\mathrm{CH}_{2}\right), 52.85(\mathrm{CH}), 54.43(\mathrm{CH}), 140.03(\mathrm{C}), 157.00(\mathrm{C}), 199.60(\mathrm{C})$; MS (GC/MS, $70 \mathrm{eV}) \mathrm{m} / \mathrm{z}$ (rel. intensity): $288\left[\mathrm{M}^{+}\right]$(75), 273 (10), 245 (10), 177 (25), 165 (21), 152 (77), 137 (100), 123 (28).

(+)-Totarol (1). To a $100 \mathrm{~mL}$ flask charged with $\mathrm{CuBr}_{2}(6.420 \mathrm{~g}, 28.75 \mathrm{mmol})$ and $\mathrm{LiBr}(1.250$ $\mathrm{g}, 14.39 \mathrm{mmol})$ under argon atmosphere was added a solution of cyclohexenone 11 (3.949 g, $13.689 \mathrm{mmol})$ prepared as described above in acetonitrile $(60 \mathrm{~mL})$ at RT. This suspension was refluxed for $20 \mathrm{~h}$, then cooled to $\mathrm{RT}$, and $\mathrm{Et}_{2} \mathrm{O}(350 \mathrm{~mL})$ and water $(500 \mathrm{~mL})$ were added. The solids were removed by filtration, and the organic layer was separated and washed with $2 \mathrm{~N} \mathrm{HCl}$ $(5 \times 200 \mathrm{~mL})$, water $(500 \mathrm{~mL})$, and dried with $\mathrm{MgSO}_{4}$. After removal of the solvent in vacuum, the crude product was purified by column chromatography over silica gel using pentane/ethyl acetate 20:1 as eluent, and then recrystallized from hexane to give (+)-totarol (1) (3.568 g, 91\%) as a yellow crystalline substance; $R_{\mathrm{f}} 0.22$ (pentane/EtOAc 20:1); $R_{\mathrm{f}} 0.36$ (pentane/EtOAc 10:1); mp $125-127^{\circ} \mathrm{C} ;[\alpha]^{20}{ }_{\mathrm{D}}+41.5\left(c 1.025, \mathrm{CH}_{2} \mathrm{Cl}_{2}\right) ;{ }^{1} \mathrm{H}$ NMR $(500 \mathrm{MHz}) \delta 0.90\left(\mathrm{~s}, 3 \mathrm{H}, \mathrm{CH}_{3}\right), 0.94$ $\left(\mathrm{s}, 3 \mathrm{H}, \mathrm{CH}_{3}\right), 1.15-1.30(\mathrm{~m}, 3 \mathrm{H}), 1.16\left(\mathrm{~s}, 3 \mathrm{H}, \mathrm{CH}_{3}\right), 1.33(2 \times \mathrm{d}$ like t with $J=6.7 \mathrm{~Hz}, 6 \mathrm{H}$, $\left.2 \mathrm{CH}_{3}\right), 1.43-1.47$ (m like br d with $\left.J=13.1 \mathrm{~Hz}, 1 \mathrm{H}\right), 1.55-1.74(\mathrm{~m}, 3 \mathrm{H}), 1.90$ (dd, $J=7.9,13.2$ $\mathrm{Hz}, 1 \mathrm{H}), 2.21$ (br d, $J=12.3 \mathrm{~Hz}, 1 \mathrm{H}), 2.70-2.77(\mathrm{~m}, 1 \mathrm{H}), 2.93$ (dd, $J=6.4,17.1 \mathrm{~Hz}, 1 \mathrm{H})$, $3.26-3.29(\mathrm{~m}, 1 \mathrm{H}), 4.40(\mathrm{~s}, 1 \mathrm{H}), 6.50(\mathrm{~d}, J=8.5 \mathrm{~Hz}, 1 \mathrm{H}), 6.98(\mathrm{~d}, J=8.5 \mathrm{~Hz}, 1 \mathrm{H}) ;{ }^{13} \mathrm{C}$ NMR $(126 \mathrm{MHz}) \delta 19.32\left(\mathrm{CH}_{2}\right), 19.46\left(\mathrm{CH}_{2}\right), 20.31\left(\mathrm{CH}_{3}\right), 21.56\left(\mathrm{CH}_{3}\right), 25.16\left(\mathrm{CH}_{3}\right), 27.12(\mathrm{CH})$, $28.75\left(\mathrm{CH}_{2}\right), 33.22(\mathrm{C}), 33.24\left(\mathrm{CH}_{3}\right), 37.67(\mathrm{C}), 39.56\left(\mathrm{CH}_{2}\right), 41.53\left(\mathrm{CH}_{2}\right), 49.53(\mathrm{CH}), 114.24$ (CH), 122.99 (CH), 130.95 (C), 133.99 (C), 143.18 (C), 151.92 (C); MS (GC/MS, 70 eV) m/z (rel. intensity): $286\left[\mathrm{M}^{+}\right]$(40), 271 (100), 243 (10), 229 (10), 215 (10), 201 (48), 189 (27), 175 (72), 159 (12), 145 (10).

\section{References}

1. For a review on bioactivities and syntheses of totarol, see: Banerjee, A. K.; Laya, M. S.; Mora, H. R.; Cabrera, E. V. Curr. Org. Chem. 2008, 12, 1050.

2. Smith, E. C. J.; Kaatz, G. W.; Seo, S. M.; Wareham, N.; Williamson, E. M.; Gibbons, S. Antimicrob. Agents Chemother. 2007, 51, 4480.

3. (a) Jaiswal, R.; Beuria, T. K.; Mohan, R.; Mahajan, S. K.; Panda, D. Biochemistry 2007, 46, 4211. (b) Constantine, G. H.; Karchesy, J. J.; Franzblau, S. G.; LaFleur, L. E. Fitoterapia 2001, 72, 572.

4. Micol, V.; Mateo, C. R.; Shapiro, S.; Aranda, F. J.; Villalaín, J. Biochim. Biophys. Acta 2001, 1511, 281.

5. Haraguchi, H.; Oike, S.; Muroi, H.; Kubo, I. Planta Med. 1996, 62, 122. 
6. Clarkson, C.; Musonda, C. C.; Chibale, K.; Campbell, W. E.; Smith, P. Bioorg. Med. Chem. 2003, 11, 4417.

7. Kubo, I.; Muroi, H.; Himejima, M. J. Nat. Prod. 1992, 55, 1436.

8. Barltrop, J. A.; Rogers, N. A. J. J. Chem. Soc. 1958, 2566.

9. Matsumoto, T.; Suetsugu, A. Bull. Chem. Soc. Jpn. 1979, 52, 1450.

10. Tada, M.; Kurabe, J.; Yasue, H.; Ikuta, T. Chem. Pharm. Bull. 2008, 56, 287.

11. (a) Marcos, I. S.; Cubillo, M. A.; Moro, R. F.; Carballares, S.; Díez, D.; Basabe, P.; Llamazares, C. F.; Benéitez, A.; Sanz, F.; Broughton, H. B.; Urones, J. G. Tetrahedron 2005, 61, 977. (b) Marcos, I. S.; Cubillo, M. A.; Moro, R. F.; Díez, D.; Basabe, P.; Sanz, F.; Urones, J. G. Tetrahedron Lett. 2003, 44, 8831.

12. Miyake, T.; Kigoshi, H.; Akita, H. Tetrahedron: Asymmetry 2007, 18, 2915.

13. Kim, M. B.; Shaw, J. T. Org. Lett. 2010, 12, 3324.

14. Das, S.; Bhattacharyya, S.; Mukherjee, D. Tetrahedron 1992, 48, 9101.

15. Evans, G. B.; Furneaux, R. H.; Gravestock, M. B.; Lynch, G. P.; Scott, G. K. Bioorg. Med. Chem. 1999, 7, 1953.

16. Löhl, T.; Markert, T.; Metz, P.; Rogachev, V. Eur. Pat. Specification 2143703 B1.

17. (a) Villamizar, J. E.; Montiel, C.; Gamez, C.; Alcalá, A.; Herrera, Y.; Salazar, F.; Tropper, E.; Canudas, N. J. Chem. Res. 2010, 34, 421. (b) van Wyk, A. W. W.; Davies-Coleman, M. T. Tetrahedron 2007, 63, 12179. (c) Nobis, M. Ger. Offen. 102005015590 A1. (d) Sarragiotto, M. H.; Gower, A. E.; Marsaioli, A. J. J. Chem. Soc. Perkin Trans. 1 1989, 559. (e) Manh, D. D. K.; Fetizon, M.; Flament, J. P. Tetrahedron 1975, 31, 1897. (f) Wenkert, E.; Majahan, J. R.; Nussim, M.; Schenker, F. Can. J. Chem. 1966, 44, 2575. (g) Grant, P. K.; Hodges, R. J. Chem. Soc. 1960, 5274. (h) Hosking, J. R. Chem. Ber. 1936, 69, 780.

18. do Céu Costa, M.; Tavares, R.; Motherwell, W. B.; Curto, M. J. M. Tetrahedron Lett. 1994, 35,8839 .

19. (a) Basabe, P.; Bodero, O.; Marcos, I. S.; Diez, D.; de Román, M.; Blanco, A.; Urones, J. G. Tetrahedron 2007, 63, 11838. (b) Zahra, J.-P.; Chauvet, F.; Coste-Maniére, I.; Martres, P.; Perfetti, P.; Waegell, B. Bull. Soc. Chim. Fr. 1997, 134, 1001. (c) Martres, P.; Perfetti, P.; Zahra, J.-P.; Waegell, B. Tetrahedron Lett. 1994, 35, 97.

20. Roldán, E. A.-M.; Santiago, J. L. R.; Chahboun, R. J. Nat. Prod. 2006, 69, 563.

21. Christenson, P. A. Tetrahedron 1988, 44, 1925.

22. (a) Kaufman, T. S.; Mischne, M. P.; Gonzalez-Sierra, M.; Ruveda, E. A. Can. J. Chem. 1987, 65, 2024. (b) Atwater, N. W. J. Am. Chem. Soc. 1960, 82, 2847.

23. Bondon, D.; Pietrasanta, Y.; Pucci, B. Tetrahedron Lett. 1977, $18,821$. 